\title{
SABERES POPULARES ACERCA DE VESPAS SOCIAIS (VESPIDAE) E ABELHAS (APIDAE) NA MATA ATLÂNTICA DO NOROESTE DO PARANÁ, SUL DO BRASIL
}

\author{
POPULAR KNOWLEDGE ABOUT SOCIAL WASPS (VESPIDAE) AND BEES (APIDAE) IN \\ THE ATLANTIC FOREST OF NORTHWEST PARANÁ, SOUTHERN BRAZIL
}

\author{
Jean Victor Nery da SILVA ${ }^{1}$; Marcos Magalhães de SOUZA²; Alex Sandro Barros de SOUZA ${ }^{1}$; José Adolfo Mota de \\ ALMEIDA $^{1}$; Hugo José MESSAGE ${ }^{3}$; Patrícia Pereira GOMES ${ }^{1 *}$
}

${ }^{1}$ Instituto Federal de Ciência e Tecnologia do Paraná, Campus Umuarama. Rodovia PR 323, KM 310 - Parque Industrial, PR, 87507-014, Paraná, Brasil; Instituto Federal de Ciência e Tecnologia de Minas Gerais, Inconfidentes, MG, Brasil; ${ }^{3}$ Universidade Estadual de Maringá, Maringá, PR, Brasil; * autor correspondente: patricia.gomes@ifpr.edu.br

\section{RESUMO}

Submitted: 01/04/2019; Accepted: 11/07/2020

Muitas espécies da ordem Hymenoptera realizam diferentes serviços ambientais e fornecem produtos de valor econômico. Apesar disso, a maioria desses insetos ainda é percebida negativamente pela população humana. Dessa forma, o objetivo deste estudo foi registrar os conhecimentos populares sobre a fauna de himenópteros (vespas sociais e abelhas) dos moradores do entorno do Parque Nacional de Ilha Grande (PNIG), na região Sul do Brasil. Para isso, foi aplicado, em outubro de 2019, um questionário semiestruturado em 50 residências no distrito de Porto Camargo, Icaraíma-PR. Para avaliar o conhecimento popular local sobre himenópteros, três modelos lineares generalizados $(\mathrm{glm}$, do inglês, General Linear Models) foram desenvolvidos. Em relação às vespas sociais, os moradores reconhecem um grande número de etnoespécies, todavia, sua relação com esses insetos não é harmônica, pois ainda é prática comum destruir seus ninhos. Em relação às abelhas, as espécies com o maior número de citações foram a exótica Apis mellifera scutellata (abelha africanizada) e a nativa Tetragonisca angustula (jataí). Em geral, os moradores não costumam retirar as colônias de abelhas, o que pode ser explicado pelo fato de reconhecerem sua importância econômica para a região. Embora os moradores possuam algum grau de conhecimento sobre vespas sociais e abelhas, isso não impede uma postura negativa para com esses insetos, sobretudo para os vespídeos. Sendo assim, é fundamental a implementação de ações de educação ambiental no sentido de reverter esse quadro e estabelecer condutas conservacionistas para esse patrimônio natural.

Palavras-chave: Etnoentomologia, etnozoologia, Conhecimentos populares, Hymenoptera, Marimbondos.

\begin{abstract}
Many species of the Hymenoptera order perform different environmental services and provide products of economic value. Despite this, most of these insects are still perceived negatively by the human population.Thus, the aim of this study was to record some popular knowledge about the hymenopterans fauna (social wasps and bees) of the Ilha Grande National Park (PNIG) residents, in the southern region of Brazil. Then, in October 2019, a semi-structured questionnaire was applied in 50 residences in the Porto Camargo district, Icaraíma-PR. To assess local popular knowledge about hymenopterans, three generalized linear models $(\mathrm{glm})$ were developed. Regarding social wasps, residents recognize a large number of ethnospecies, however, their relationship with these wasps is not harmonious, as it is still common practice to destroy their nests. Regarding bees, the species with the highest number of citations were the exotic Apis mellifera scutellata (Africanized bee) and the native Tetragonisca angustula (jataí). In general, residents do not usually remove bee colonies, which can be explained by the fact that they recognize their economic importance for the region. Although the residents have some degree of knowledge about social wasps and bees, this does not prevent a negative attitude towards these insects, especially for the hornets. Therefore, it is essential to implement environmental education actions in order to reverse this situation and establish conservationist conducts for this natural heritage.
\end{abstract}

Keywords: Ethnoentomology, ethnozoology, Popular knowledge, Hymenoptera, Wasps. 


\section{INTRODUÇÃO}

A maneira como a humanidade interage com os recursos naturais disponíveis, acumulando conhecimentos sobre eles e explorando-os economicamente, parece ser característica comum a todas as sociedades em diferentes culturas (LOPES et al., 2010). As diversificadas formas de interação entre os seres humanos e outros animais, por exemplo, são valiosas não apenas porque proporcionam benefícios utilitários e econômicos, mas também porque foram incorporados ao senso de pertencimento ao meio e às mais diversas práticas culturais (ALVES e SOUTO, 2015; ALVES e ALBUQUERQUE, 2018). Essas interações deram origem a um campo de conhecimento que resultou na disciplina etnobiologia (LOPES et al., 2010).

Considerando que as relações entre os humanos e os demais animais podem variar dependendo de aspectos econômicos, geográficos e culturais, cada espécie tem sua importância para cada sociedade. Esse é o caso do filo Arthropoda, por exemplo, que representa o maior grupo de organismos vivos no planeta, constituindo aproximadamente $85 \%$ das espécies animais, sendo mais de um milhão delas descritas (BRUSCA e BRUSCA, 2007; GHOSH e SATPUTE, 2016), embora se estime que existam milhares de espécies ainda não catalogadas.

A espécie humana se beneficia, direta ou indiretamente, de muitos dos artrópodes, tais como insetos e crustáceos, como fonte de alimento, controle biológico de pragas, na produção de mel, para experimentos científicos, e por meio da polinização realizada por muitos desses animais, a qual é fundamental para a agricultura (ALMEIDA e CAVICHIOLI, 2006; GULLAN e CRANSTON, 2017). No entanto, as transformações que a espécie humana vem promovendo nos ambientes naturais em virtude da expansão das áreas agrícolas e urbanas, têm gerado profundas alterações nas funções ecossistêmicas (BARNOSKY et al., 2011; CLARK e TILMAN, 2017). Essa transformação contínua tem impactos diretos na entomofauna, uma vez que apenas um pequeno número de espécies é capaz de sobreviver e se desenvolver em ecossistemas alterados (OLIVEIRA et al., 2017).

Apesar de serem fundamentais para o funcionamento dos ecossistemas e estarem entre os grupos de animais com os quais o ser humano mantém estrita relação, a maioria dos insetos ainda é percebida negativamente pela população humana (ULYSSÉA et al., 2010; BOMFIM et al., 2016). Dentre eles, a ordem Hymenoptera está entre as três maiores em número de espécies animais, com aproximadamente 250.000 espécies (COSTA et al., 2011). No entanto, o conhecimento popular sobre himenópteros (vespas e abelhas) ainda apresenta uma visão distorcida sobre esses importantes insetos, sendo suas colônias geralmente destruídas quando avistadas (PÁDUA et al., 2017).

As comunidades tradicionais atribuem aos insetos diferentes funções, algumas consideradas benéficas (abelhas) e outras consideradas maléficas (vespas ou marimbondos) (BOMFIM, et al., 2016). No caso das abelhas, os conhecimentos populares geralmente associam esses insetos a usos medicinais, alimentícios, cosméticos e na fabricação de utensílios (MODRO et al., 2009). Esses conhecimentos tradicionais sobre as abelhas, de certa forma, corroboram com a conservação desses insetos por meio da percepção que as comunidades tradicionais têm sobre eles (FERREIRA et al., 2010).

De acordo com Bolzani et al. (2012), o Brasil abriga uma rica diversidade biológica, além de ser um país multicultural, com uma rica sociodiversidade, representada por povos indígenas e inúmeras comunidades locais e/ou tradicionais, as quais reúnem um amplo conhecimento do uso tradicional da biodiversidade (DIEGUES et al., 1999; BERTOLDI, 2014). Nesse sentido, a pesquisa na área de etnozoologia vem se intensificando nos últimos anos e o Brasil tem se tornado uma importante fonte dessa produção científica (ALVES e SOUTO, 2011).

No que diz respeito à etnoentomologia, a literatura reporta interações culturais com diferentes táxons de insetos (ULYSSÉA et al., 2010; SANTOS et al., 2015), seja no âmbito cultural, na literatura, música, arte e nas crenças religiosas de diferentes etnias (MACHADO, 1987; COSTANETO, 2000, 2004b; COSTA-NETO e CARVALHO, 2000; SOUZA e ZANUNCIO, 2012). No Brasil, a maior parcela das pesquisas etnozoológicas com insetos têm se concentrado na região Nordeste (COSTA-NETO, 1998, 2004a, 2004b, 2004c, 2013; SILVA e PAZ, 2012; CARVALHO et al., 2014; BONFIM et al., 2016). Alguns estudos foram realizados na Amazônia, região Norte do país (SANTOS e ANTONINE, 2008; PETIZA et al., 2013; ATHAYDE et al., 2016) e outros na região Centro-Oeste (MODRO et al., 2009; FERREIRA et al., 2010; SANTOS et al., 2015). Nas regiões Sudeste e Sul, os estudos de Etnozoologia são escassos, podendo ser citados os trabalhos de Pádua et al. (2017) em Minas Gerais, e Ulysséa et al. (2010), em Santa Catarina. 
Conhecer os conceitos etnozoológicos de comunidades locais, com pouca informação disponível sobre a sua interação com insetos, como é o caso do presente estudo, é fundamental para promover a interação entre o conhecimento popular e o científico. Dessa forma, o objetivo deste estudo foi registrar conhecimentos populares sobre a fauna de himenópteros (vespas sociais e abelhas) dos moradores do entorno do Parque Nacional de Ilha Grande (PNIG), na região Sul do Brasil.

\section{MATERIAL E MÉTODOS}

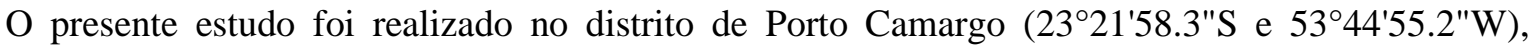
município de Icaraíma, localizado no Noroeste do Estado do Paraná, Brasil (Figura 1). O Bioma predominante dessa região é a Mata Atlântica, a qual apresenta fisionomia de mata ombrófila, fitogeografia de floresta estacional semidecidual e fitofisionomia herbácea de influência flúviolacustre (SLOVENSKI et al., 2008).

A comunidade foi selecionada por estar localizada no entorno do Parque Nacional de Ilha Grande (PNIG), bem como por abrigar moradores que viveram boa parte de suas vidas nas ilhas do Parque, as quais foram desapropriadas por motivos anteriores à implementação dessa Unidade de Conservação (UC): cheias no rio Paraná e a construção prevista de uma hidrelétrica na região, embora esta não tenha sido implementada. Todavia, alguns moradores ainda dependem dessa UC para sua subsistência, como os apicultores que possuem caixas de abelhas nas ilhas do Parque.
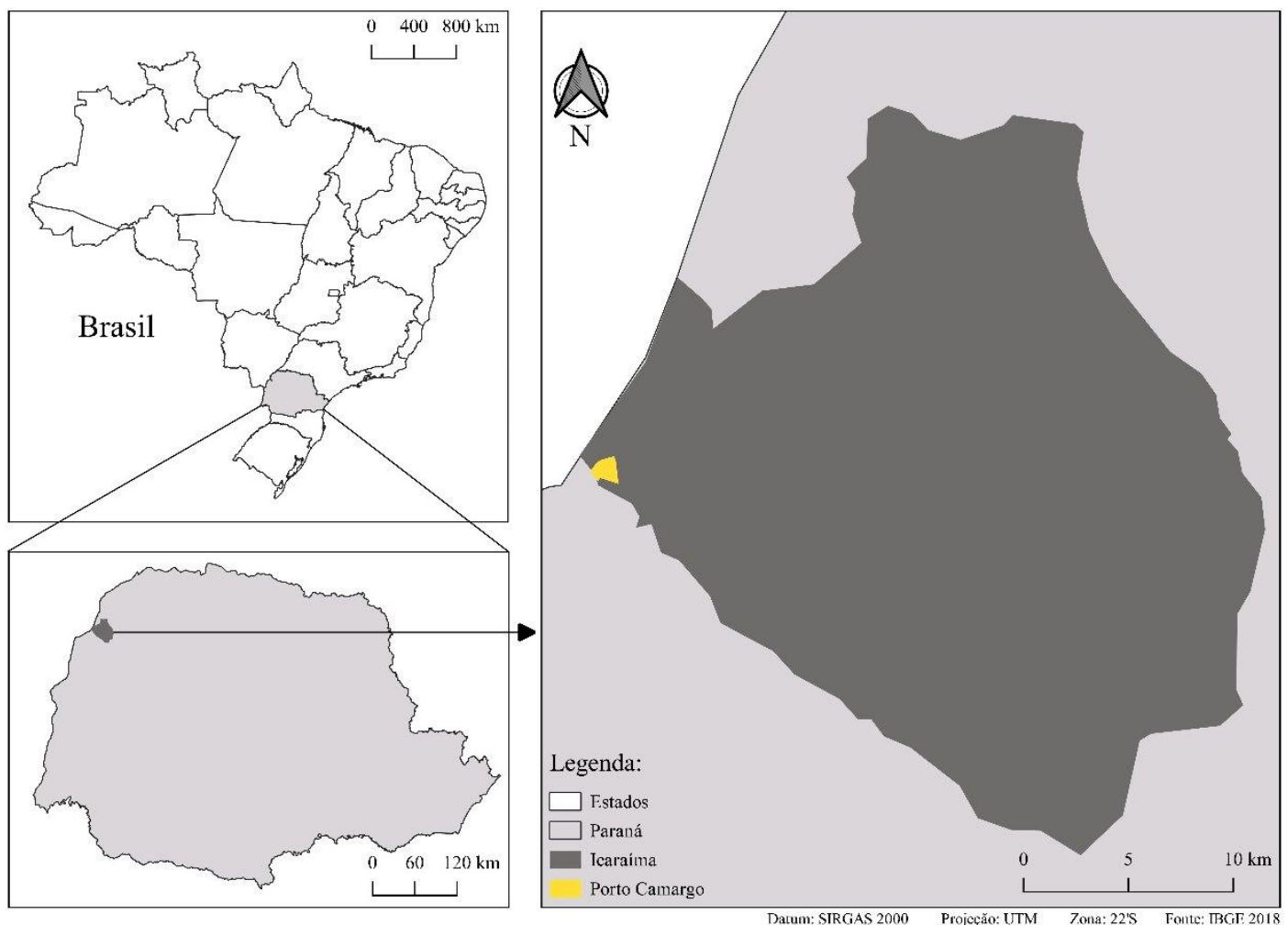

Figura 1: Localização do distrito de Porto Camargo, Icaraíma-PR (Fonte: IBGE, 2018).

O levantamento de informações sobre os saberes populares dos moradores de Porto Camargo acerca dos himenópteros (vespas sociais e abelhas) contou com o apoio da Prefeitura Municipal de Icaraíma, por meio da Vigilância Municipal de Saúde, que contribuiu com a delimitação amostral e com apoio logístico para a aplicação dos questionários. O distrito possui cerca de 556 imóveis e aproximadamente 781 habitantes. Ao longo deste estudo, foram realizadas 50 entrevistas, em 50 residências, o que corresponde a aproximadamente $8,99 \%$ das residências existentes no distrito.

A pesquisa foi realizada por meio de metodologia exploratória, descritiva e interpretativa, e abordagem quali-quantitativa a partir da organização de lista com as etnoespécies citadas (PRODANOV e FREITAS, 2013). Os indivíduos foram entrevistados individualmente, sendo as perguntas e os diálogos direcionados pelo entrevistador. Quando os entrevistados expressavam suas ideias, para além do que estava estabelecido no questionário, essas importantes informações adicionais eram também registradas. 
Para analisar as entrevistas, empregou-se análise de conteúdo (BARDIN, 2011), que constitui uma forma de tratar dados em pesquisas qualitativas e quantitativas utilizando métodos de análise baseados em organização, codificação, categorização, inferência e informatização das análises. Esse método foi usado pela primeira vez nos Estados Unidos no início do século XX como um instrumento de análise da área de comunicações, sendo aperfeiçoado por diversos autores ao longo dos anos (SANTOS, 2012). A análise de conteúdo se baseia em pressupostos de uma concepção crítica e dinâmica da linguagem. Nessa linha, a linguagem é considerada uma construção real de toda a sociedade trazendo marcas de suas práticas culturais. Assim, a análise de conteúdo pode ser compreendida como uma metodologia que evita uma visão formalista da linguagem, funcionando sem atribuir um valor exagerado às palavras e concentrando o foco nas análises dos aspectos semânticos (FRANCO, 2005).

As entrevistas foram realizadas no sentido de compreender a percepção da comunidade sobre a fauna de himenópteros: como os classificam com base nas suas características morfológicas, comportamentais e ecológicas; se apreciam ou não a presença desses insetos perto de suas residências; se fazem uso desses insetos como alimento, remédio etc.; e se compreendem a sua importância ambiental.

Antes de cada entrevista, os participantes receberam um Termo de Consentimento Livre e Esclarecido, fornecendo informações sobre a natureza e os objetivos do estudo. Foram solicitadas assinaturas do entrevistado, conforme exigido pela legislação vigente (Resolução $\mathrm{n}^{\mathbf{0}}$ 466, de 12/1012/2012, Conselho Nacional de Saúde). Este estudo foi autorizado pelo Comitê Nacional de Ética em Pesquisa (Comissão Nacional de Ética em Pesquisa - CONEP) com Certificado de Apresentação para Consideração Ética $n^{\circ} 16746619.0 .0000 .8156$. Não foram realizadas coletas de espécimes de abelhas e vespas a fim de identificar as espécies, pois isso não contemplou o objetivo do presente estudo, todavia utilizou-se o trabalho de Pádua et al. (2017) como referência para definição de alguns gêneros.

A aleatorização dos locais de amostragem foi realizada a partir de uma adaptação do método LIRAa - Levantamento Rápido de Índices de Infestação pelo Aedes aegypti ou Índice de Breatea, que é utilizado pelo Ministério da Saúde para determinar qual a quantidade de casas de um quarteirão deve ser avaliada para se obter a densidade larvária de A. aegypti (BRASIL, 2005). No presente estudo, esse método foi utilizado somente para determinar quais casas seriam visitadas. Sendo assim, foram separados seis quarteirões aleatórios entre os bairros do distrito de Porto Camargo, sendo selecionadas 50 residências.

As entrevistas foram realizadas por meio de questionários semiestruturados com o objetivo de registrar os conhecimentos e as percepções dos moradores sobre vespas sociais e abelhas (ver Questionário no Apêndice). As porcentagens obtidas não correspondem exatamente ao número de entrevistas, pois a maioria dos entrevistados deu mais de uma resposta para a mesma pergunta.

Para avaliar o conhecimento popular local sobre himenópteros, três modelos lineares generalizados ( $\mathrm{glm}$, do inglês General Linear Models) foram desenvolvidos usando a função 'glm' do software R. Dois modelos foram feitos com a variável resposta de contagem. No primeiro, a variável resposta foi o número de tipos de marimbondos $(1,2,3,4,5$ e 6$)$ conhecidos pelos populares; no segundo modelo a variável foi o número de tipos de abelhas $(1,2,3,4$ e 5); o terceiro modelo foi gerado com uma variável resposta binária, a atitude em relação ao ninho (pergunta: "já tirou ninho?"; resposta: sim=1 ou não=0). Para os três modelos, as variáveis preditoras analisadas foram a idade (discreta, de 18 a 75 anos), o tempo de residência na cidade (discreta, de 1 a 56 anos) e o sexo (binária, masculino e feminino) do entrevistado; as interações entre essas variáveis também foram testadas. Antes de gerar os modelos concorrentes, foram realizadas análises exploratórias dos dados de acordo com Zuur et al. (2010).

Dessa forma, a distribuição das variáveis preditoras de contagem (tipos de marimbondos e de abelhas) foi identificada por histogramas (função 'hist'). A homocedasticidade foi testada pelo teste de Levene ( $p>0,05$; função 'leveneTest', pacote 'car', FOX e WEISBERG, 2019) e a normalidade dos resíduos das variáveis resposta foi testada pelo teste de Shapiro-Wilk ( $\mathrm{p}>0,05$; função 'shapiro.test'). A multicolinearidade foi testada calculando o Valor de Inflação da Variância (GVIF, do inglês Generalized Variance Inflation Factor) pela função 'corvif' e a função de suporte 'HighstatLibV11.R'. A linearidade das variáveis resposta foi testada pela função 'chart.Correlation' do pacote 'PerformanceAnalytics' (PETERSON e CARL, 2020). A autocorrelação para a variável resposta de cada modelo foi estimada pela função 'acf'. Nenhuma variável precisou ser excluída. 
Nos glm, para prever o número conhecido de tipos de marimbondos e de abelhas, os modelos foram gerados assumindo a distribuição de Poisson dos resíduos e a função-de-ligação log após a confirmação do ajuste da variável resposta usando as funções 'fitdist', 'descdist' (ambas do pacote 'fitdistrplus', DELIGNETTE-MULLER e DUTANG, 2015) e 'goodfit' (pacote vcd, MEYER et al., 2006). Ainda no $\mathrm{glm}$, mas agora para prever a atitude em relação ao ninho, o modelo foi gerado assumindo a distribuição Binomial e a função-de-ligação logit, sem necessidade de checagem.

Os VIF foram checados em cada um dos três modelos selecionados (um para cada variável resposta) pela função 'vif' (pacote 'car'). A quantidade de variância explicada por cada modelo, também chamada de resíduos ou desvio de cada modelo, foi obtida diretamente pela função 'summary', sendo a significância $(\mathrm{p}<0,05)$ de cada variável preditora. $\mathrm{O}$ valor de $D^{2}$ ajustado foi calculado usando a função 'Dsquared' (pacote 'modEvA', BARBOSA et al., 2016). Para cada variável resposta, foram gerados $\mathrm{glm}$ com todas as combinações possíveis entre as variáveis preditoras. $\mathrm{O}$ critério de Akaike (AIC) foi usado para selecionar os modelos (SYMONDS e MOUSSALLI, 2011) em conjunto com o valor dos resíduos. Esse protocolo analítico foi desenvolvido a partir de Silva et al. (2019).

\section{RESULTADOS E DISCUSSÃO}

Durante o presente estudo, observou-se que a maioria dos moradores (76\%) foi capaz de diferenciar as vespas das abelhas, respondendo que a abelha é "mais gordinha" e o marimbondo "mais comprido", referindo-se ao formato do abdômen desses himenópteros. Outra característica também relatada para diferenciar esses insetos está relacionada à produção de mel: "A abelha produz mel e o marimbondo não".

Quando os moradores foram questionados se conheciam algum tipo de marimbondo, observou-se que os mesmos ficavam preocupados em não saber o nome científico das espécies. Todavia, ao reformular as perguntas e pedir para que falassem os nomes populares, notou-se que alguns moradores conhecem os marimbondos por algum nome tradicional, mas também por suas características morfológicas e coloração. Foram reportadas 28 etnoespécies de vespas sociais (Tabela 1), reconhecidas pela forma do ninho ou comportamento, resultado ligeiramente maior que o observado por Pádua et al. (2017), em que os entrevistados utilizaram 24 nomes populares para se referir a esses insetos.

As etnoespécies mais citadas foram marimbondos-exu (Brachygastra spp.) e marimbondovermelho ou caboclo (Polistes spp.). De forma generalista, isso permite identificar no máximo o táxon do gênero, como exu-do-pasto, que remonta à sua agressividade e hábito de construção de suas colônias no pasto, sendo este um termo comumente utilizado para o gênero Brachygastra (SOUZA e ZANUNCIO, 2012). Ou no caso do marimbondo-chapéu, que pela forma arquitetônica do ninho correlaciona-se com espécies do gênero Apoica, sendo que em outros Estados do Brasil o gênero é conhecido como caba-chapéu ou marimbondo-branco (COSTA-NETO, 2004c; SOUZA et al., 2008). É possível também reconhecer algumas espécies em função da forma peculiar dos ninhos, como é o caso do marimbondo-casca-de-tatu, gênero Synoeca (SOUZA et al., 2008).

Tabela 1: Espécies de vespas citadas na entrevista e identificação dos possíveis táxons.

\begin{tabular}{lll}
\hline Etnoespécie & Citações (\%) & Possível táxon \\
\hline Marimbondo-exu ou exu-de-pasto & $19 \%$ & Brachygastra spp. \\
Marimbondo-vermelho ou caboclo & $18 \%$ & Polistes spp. \\
Marimbondo-cavalo & $8 \%$ & Polistes spp. \\
Marimbondo-amarelo & $5 \%$ & \\
Marimbondo-amarelinho & $5 \%$ & \\
Marimbondo-tatu ou casca-de-tatu & $5 \%$ & Synoeca spp. \\
Marimbondo-preto & $4 \%$ & \\
Marimbondo-amarelo-e-preto & $4 \%$ &
\end{tabular}




\begin{tabular}{|c|c|c|}
\hline Etnoespécie & Citações (\%) & Possível táxon \\
\hline Marimbondo-caçador-de-aranha & $4 \%$ & Pompilidae/Sphecidae \\
\hline Magangaba* & $2 \%$ & Xylocopa spp. \\
\hline Marimbondo-preto-pequeno & $2 \%$ & \\
\hline Marimbondo-chapéu & $2 \%$ & Apoica spp. \\
\hline Marimbondo-perdiz & $2 \%$ & \\
\hline Marimbondo-cego & $2 \%$ & \\
\hline Marimbondo-tapa-guela & $2 \%$ & Synoeca spp. \\
\hline Marimbondo-cachorra & $2 \%$ & \\
\hline Marimbondo-de-luz & $2 \%$ & Apoica spp. \\
\hline Marimbondo-tremedeira & $1 \%$ & \\
\hline Marimbondo-mandassaia & $1 \%$ & Melipona spp. \\
\hline Marimbondo-pequeno & $1 \%$ & \\
\hline Marimbondo-vermelho-e-preto & $1 \%$ & \\
\hline Marimbondo-preto que poliniza maracujá & $1 \%$ & \\
\hline Marimbondo-marrom & $1 \%$ & \\
\hline Marimbondo-rajadinho & $1 \%$ & \\
\hline Arapuá* & $1 \%$ & Trigona spinipes \\
\hline Marimbondo-amarelo-listrado & $1 \%$ & \\
\hline Marimbondo-calunga & $1 \%$ & \\
\hline Marimbondo-vaqueiro & $1 \%$ & \\
\hline
\end{tabular}

*A espécie citada não pertence ao grupo das Vespas.

O número de etnoespécies de marimbondos citadas reflete uma relação próxima das pessoas com esses insetos (PÁDUA et al., 2017), o que fica evidenciado quando reconhecem as formas dos ninhos e os comportamentos desses insetos. Apesar dessa proximidade, a relação das pessoas com os marimbondos não é harmônica, pois ainda é prática comum destruir seus ninhos, como reportado por $68 \%$ dos entrevistados, que costumam utilizar fogo e inseticida spray, além de utilizarem vassoura, produtos de limpeza e até mesmo jogar pedras nos ninhos. Um dos entrevistados respondeu: "Retiro o ninho só quando está na passagem de crianças e idosos". Além disso, diversas pessoas alegam, equivocadamente, que as vespas não são boas ou não possuem importância, o que reflete negativamente sobre a conservação desses insetos. Devido a esta percepção negativa sobre as vespas, os moradores em geral retiram ou destroem seus ninhos. Pádua et al. (2017) também observaram que poucos entrevistados citaram algum benefício das vespas, mesmo que de maneira equivocada na maioria das vezes. Isso reflete negativamente as populações desses insetos e, por consequência, diminui o acesso aos serviços ambientais prestados pelas vespas sociais, como controle biológico (PREZOTO et al., 2019) e polinização (HERMES e KÖHKER, 2006; CLEMENTE et al., 2013). Isso é preocupante, pois esse fato, somado aos processos de desmatamento e fragmentação crescentes dos ambientes naturais, promove a redução da riqueza e abundância desses insetos (GRAÇA e SOMAVILLA, 2018; BUENO et al., 2019). Todavia, não há dados suficientes para inferir sobre o grau de ameaça de extinção de espécies, embora já haja indicações de áreas consideradas relevantes para a conservação das populações de vespas sociais em áreas de Mata Atlântica no Brasil (SOUZA et al., 2020). Nesse âmbito, os estudos de etnoentomologia permitem uma análise das relações 
estabelecidas entre as populações humanas e a biota, o que é relevante para definir estratégias que possibilitem a conservação dos recursos naturais (COSTA-NETO, 2012).

Sobre os locais de construção dos ninhos ("caixas") dos marimbondos, $42 \%$ dos entrevistados responderam que eles são feitos em ambientes urbanizados e naturais, enquanto $36 \%$ disseram que encontram os ninhos somente em ambiente urbanos, mas $14 \%$ afirmaram que os ninhos são encontrados somente em ambientes naturais e $8 \%$ não souberam responder. Os resultados obtidos a partir das entrevistas mostram que os moradores percebem que muitas espécies de vespas sociais são encontradas em ambientes naturais e urbanos, construindo seus ninhos próximos às habitações humanas, um sinal de sinantropia. De acordo com a literatura (PREZOTO et al., 2007; OLIVEIRA et al., 2017), algumas espécies de vespas sociais preferem a construção de seus ninhos em ambientes urbanos, principalmente por causa da pressão da urbanização sobre os ambientes naturais, reduzindo essas áreas; devido a sua estratégia de sobrevivência, já que as construções humanas podem funcionar como abrigo contra intempéries climáticas, além de reduzir a competição com outras espécies.

Quando questionados sobre uso de vespas sociais na alimentação ou produção de remédios, quase a totalidade dos moradores de Porto Camargo (96\%) respondeu que esses insetos não são utilizados com finalidade medicinal e nem alimentícia. Somente $4 \%$ disseram que os utilizam como isca ou iguaria para aromatizar aguardente.

Identificou-se também o uso de simpatias, que segundo os entrevistados funcionam tão bem quanto remédios. Em uma das simpatias, quando o indivíduo recebe uma ferroada, ele deve cuspir no chão e pisar com o pé em cima e arrastar para trás ("Eu faço uma simpatia, cuspo no chão e arrasto o pé em cima"). Outro exemplo de simpatia, que foi a mais citada, é colocar algum material de aço (faca, colher etc.) em cima do local afetado e manter lá até reduzir a dor. De acordo com um dos entrevistados, "quando levo picada de marimbondo coloco aço em cima da pele, que a pele vai puxar a química do aço, e vai no veneno parando que faz parar de doer".

Em relação às abelhas, as espécies com o maior número de citações foram a exótica (já africanizada no país) Apis mellifera scutellata, conhecida como abelha europa (32\%) e a nativa Tetragonisca angustula, conhecida como abelha jataí (30\%). Apenas 2\% dos entrevistados responderam que não conhecem nenhuma espécie de abelha (Tabela 2). As duas espécies mais citadas neste estudo são mais conhecidas em função do seu valor econômico, o que as tornam importantes para a região. Os produtos extraídos das abelhas fornecem renda para as pessoas que residem no distrito e para os apicultores da região, sendo que um morador enfatizou que "o mel de abelha jataí produzido nas ilhas é o melhor mel do Paraná". O mel de abelhas possui alto valor econômico, embora sua qualidade possa ser influenciada pela região em que estão as colmeias, por possíveis chances de contaminações por fungos e bactérias, e até mesmo em função do risco de contaminação durante o processo de beneficiamento dos produtos, o que pode resultar na redução da pureza do mel (BRAGHINI et al., 2017).

Além disso, a espécie exótica A. m. scutellata é muito comum para esses moradores do entorno do Parque Nacional de Ilha Grande, já que muitos deles possuem muitas colônias dessa espécie instaladas dentro do parque. Esta espécie foi introduzida no Brasil a partir da Europa e África, e atualmente, responde pela maior parte do mel produzido no país (LOPES et al., 2005). No caso das abelhas nativas os nomes populares dos meliponíneos são muito diversos, o que pode ser explicado pelas variações linguísticas regionais, gerando casos em que o mesmo nome pode representar mais de uma espécie diferente de abelha (NOGUEIRA-NETO, 1997; SILVA e PAZ, 2012).

Tabela 2: Espécies de abelhas citadas na entrevista e identificação dos possíveis táxons.

\begin{tabular}{lcl} 
Etnoespécie & Citações $(\boldsymbol{\%})$ & Possível táxon \\
\hline Europa & $32 \%$ & Apis mellifera scutellata \\
Jataí & $30 \%$ & Tetragonisca angustula \\
Arapuá & $13 \%$ & Trigona spinipes \\
Africana & $9 \%$ & Apis mellifera scutellata \\
Mumbuca ou caga-fogo & $3 \%$ & Oxytrigona spp.
\end{tabular}




\begin{tabular}{lcl}
\hline Etnoespécie & Citações $(\boldsymbol{\%})$ & Possível táxon \\
\hline Mamangava & $3 \%$ & Xylocopa spp. \\
Europa sem ferrão & $2 \%$ & \\
Europa africanizada & $2 \%$ & Apis mellifera scutellata \\
Caçununga * & $1 \%$ & Agelaia ou Mischocyytarus spp. \\
Mirim & $1 \%$ & Plebeia spp. \\
Mandaguari & $1 \%$ & Scaptotrigona postiça \\
Cumunçu & $1 \%$ & \\
Abelha "normal" Europa & $1 \%$ & Apis mellifera scutellata \\
Sanharó & $1 \%$ & Trigona spp. \\
\hline
\end{tabular}

*A espécie citada não pertence ao grupo das abelhas e sim uma vespa social.

Sabe-se que interação entre as abelhas e plantas garantiu aos vegetais o sucesso na polinização cruzada (SANTOS, 2010). Essa interação é fundamental para a manutenção da biodiversidade por meio dos serviços prestados às comunidades florísticas de todo o mundo via polinização (RÊGO e ALBUQUERQUE, 2006). Neste contexto, o ser humano também é beneficiado, já que ao longo dos anos desenvolveu técnicas que lhe permitiram tirar proveito do trabalho de polinização das abelhas, sendo que a diminuição desse processo pode inclusive afetar a produção de alimentos no mundo (SOUZA et al., 2007; OLLERTON et al., 2011; SILVA e PAZ, 2012). Dessa forma, as populações humanas apresentam uma percepção positiva em relação às abelhas, em especial com as abelhas sem ferrão (REYES-GONZÁLEZ et al., 2014).

Os meliponíneos são encontrados em regiões tropicais e subtropicais, distribuindo-se amplamente na maior parte do território Latino-Americano (NOGUEIRA-NETO, 1997; SILVA e PAZ, 2012). No Brasil são conhecidas mais de 400 espécies de abelhas sem ferrão, que apresentam heterogeneidade na cor, tamanho, forma, hábitos de nidificação e população dos ninhos (SANTOS, 2010). Além de não causarem nenhum dano às pessoas, como ocorre com A. m. scutellata e vespas, delas se obtêm produtos e subprodutos bastante valorizados economicamente, tais como, mel, pólen, própolis e geoprópolis (SILVA e PAZ, 2012; REYES-GONZÁLEZ et al., 2014). No presente estudo, os moradores demonstraram ter maior conhecimento para falar desses insetos, especialmente ao ressaltar sua importância na produção de mel, própolis e polinização, relatando, por exemplo, que " $a$ abelha tem a importância do mel".

A maioria (70\%) dos entrevistados respondeu que fazem uso de produtos provenientes das abelhas, como cera, mel e própolis (Fig. 2). Segundo eles, o produto mais utilizado é o mel, sendo usado como remédio na forma de xarope; puro ou misturado com limão para combater gripe e bronquite; como alimento, para adoçar café e comer com pão. No presente estudo, a própolis também é usada como remédio para gripe e para dor de garganta. Alguns moradores citaram que usam a cera para passar no cabo de vassoura e assim não machucar a mão. 


\section{Uso de Abelhas}

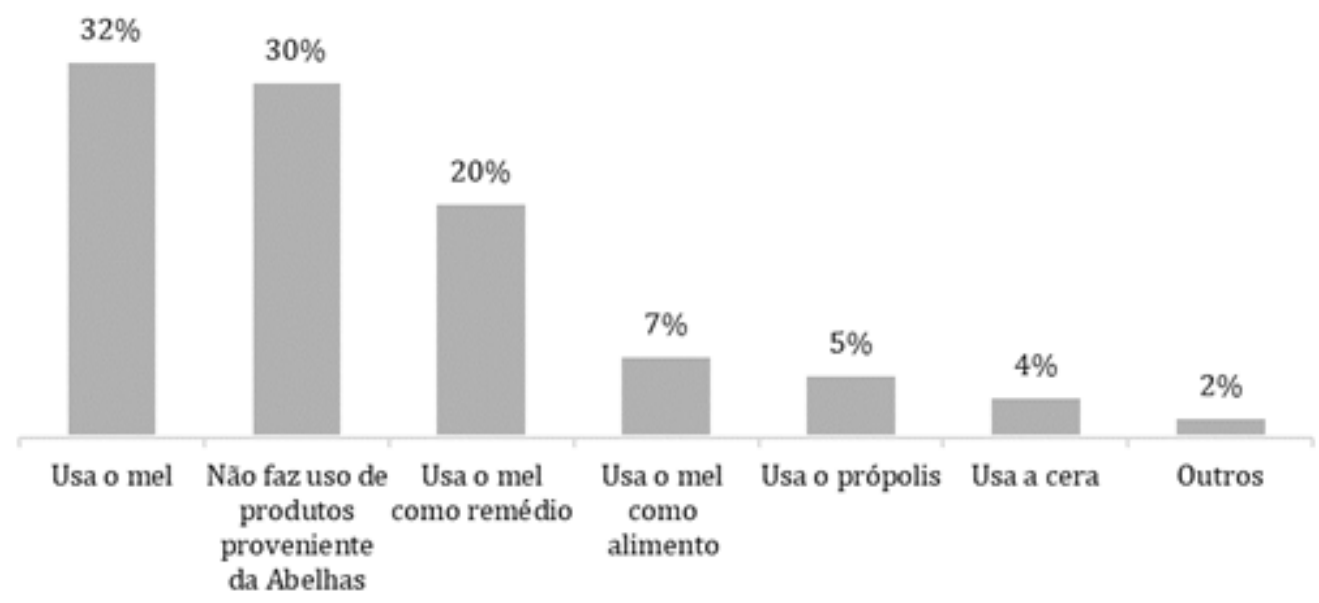

Figura 2. Uso de produtos provenientes das abelhas pela população de Porto Camargo, Icaraíma, PR.

Todavia, apesar da importância ecológica e econômica do meliponíneos, tem-se observado um declínio na riqueza dessas espécies de abelhas nativas, o que pode ser causado pelo aumento da abundância de colônias de A. $m$. scutellata, desmatamento das florestas nativas, práticas incorretas de extração dos produtos apícolas, mudanças climáticas e uso de defensivos agrícolas (LOPES et al., 2005; SANTOS, 2010; REYES-GONZÁLEZ et al., 2014).

No Brasil, 17 espécies de abelhas correm algum risco de extinção (ICMBIO, 2018). Dentre as espécies listadas com algum risco de extinção, cinco foram citadas pelos moradores de Porto Camargo, o que mostra a necessidade urgente de evitar a destruição das colônias, reduzir o uso de defensivos agrícolas na região (THOMPSON, 2003; ROCHA e ALENCAR, 2012), bem como promover a manutenção dos ambientes naturais (MORATO e CAMPOS, 2000; MENEZES et al., 2007), a fim de assegurar a proteção da diversidade biológica.

Todavia, o conhecimento sobre as espécies ameaçadas de extinção no Estado do Paraná ainda permanece insatisfatória. Dentre as 18 espécies de abelhas citadas por Schwartz-Filho et al. (2004) no livro vermelho da fauna em extinção do estado, apenas 17 localidades possuem registros de distribuição. Este fato indica que muitas regiões do estado permanecem ainda inexploradas, pois o gênero Oxytrigona está listado tanto na lista estadual quanto na nacional e foi citado por cerca de 3\% dos moradores entrevistados, sendo que a localidade de estudo não aparece listada no livro, mesmo fazendo parte do entorno de uma Unidade de Conservação: o Parque Nacional Ilha Grande.

Em relação aos ninhos de abelhas, $57 \%$ dos entrevistados afirmaram que esses insetos constroem suas colônias em ambientes naturais, 18\% disseram que as abelhas ocupam espaços naturais e urbanos, enquanto $14 \%$ responderam que as abelhas gostam de ambientes urbanos e $8 \%$ não sabem onde ficam suas colônias. Observou-se ainda que, embora as abelhas sejam insetos bastante comuns, $2 \%$ dos entrevistados desconhecem os locais em que elas estabelecem seus ninhos. Sobre seus ninhos, os entrevistados disseram que elas costumam fazê-los em buracos, tanto em ambientes naturais como árvores e ocos de pau, quanto em ambientes urbanos, aproveitando frestas e aberturas nas construções humanas, bem como em caixas utilizadas para apicultura. Isso pode ser devido à maior proteção desses locais contra agentes externos e predadores. De acordo com Morato (2000), a mudança das abelhas para os ambientes urbanos pode ser devido à ausência de fontes de alimentos em seus hábitats naturais ou ao aumento da competição pelos mesmos recursos contra uma espécie mais eficiente na captação dos mesmos, ocasionando a redução das populações desses indivíduos nos ambientes naturais.

Além disso, $68 \%$ dos entrevistados não adotam nenhuma prática de retirada das colônias de abelhas e $32 \%$ deles, quando o fazem, utilizam algum método de manejo, como o uso de uma caixa para retirar o enxame ou o uso de macacão de apicultura para o manejo. Ou então chamam os apicultores da região para fazerem o manejo e a destinação correta dos ninhos e das abelhas. A retirada dos ninhos de abelhas por medo de acidentes foi citada em algumas entrevistas, já que muitas pessoas são alérgicas às substâncias inoculadas por esses insetos. De qualquer modo, por mais que medidas preventivas sejam executadas durante a retirada dos ninhos e colônias de himenópteras, a probabilidade de sofrer 
um acidente, ou seja, "levar uma ferroada de abelha ou marimbondo" é muito grande. Além disso, acidentes com himenópteros são comuns entre os pescadores da região, que se deparam com enxames de abelhas, por exemplo, enquanto navegam no rio Paraná. Nesse caso, a medida preventiva adotada por eles é pular na água e/ou afugentar os enxames jogando barro e água.

Apesar disso, no presente estudo, em geral os entrevistados não costumam retirar as colônias de abelhas, o que pode ser explicado pelo fato de reconhecerem sua importância econômica para a região. Isso fica evidente pelo fato de que quando necessitam retirar uma colônia, os moradores chamam os apicultores da região para fazerem o manejo e a destinação correta dos ninhos e das abelhas. De acordo com a Coordenadoria de Educação Ambiental de São Paulo (2013), no caso de haver necessidade de retirada de colmeias e vespeiros, recomenda-se que estes devem ser manipulados somente por pessoas que conhecem os riscos envolvidos bem como sua forma correta de manejo, além de estabelecer uma área de isolamento para evitar acidentes a pessoas e animais. Além disso, é necessário também que a pessoa que manipular os ninhos esteja utilizando o equipamento de apicultura completo.

Todos os moradores entrevistados disseram ainda que já sofreram acidentes com abelhas, mas que em geral não fazem nada. "Só espero a dor passar", disseram cerca de $31 \%$ deles. Além disso, também se ouviu que "Só procuro passar algo ou ir ao médico quando levo várias ferroadas por enxames de abelhas". Algumas pessoas relataram, inclusive, que só descobriram que eram alérgicas a partir desses acidentes, sendo que em muitos casos tiveram que comparecer a um hospital para serem medicadas. Todavia, $69 \%$ dos entrevistados disseram que fazem uso de alguma terapia alternativa para reduzir a dor sentida no local da ferroada e/ou para reduzir o inchaço. Em geral, utilizam itens encontrados comumente em casa, como álcool, gelo, gel para massagem muscular, pomadas e água gelada. Também fazem uso de medicamentos direcionados a outros tratamentos, como Vick Vaporub®, dipirona, antialérgicos e analgésicos, sendo que muitos entrevistados se auto medicam, sem prescrição médica.

O conhecimento popular local sobre as espécies de marimbondos, de abelhas e a atitude das pessoas quando os encontram, foi influenciado por uma combinação da idade, do tempo que reside na cidade e do sexo. Os glm sugeriram associações significativas entre o número conhecido de tipos de marimbondos e de abelhas e a atitude em retirar ou não um ninho - a partir daqui chamados de GLMmarimbondo, GLмabelha, GLmninho - com as variáveis preditoras selecionadas (Tabela 3). A variância explicada por GLmmarimbondo (tipos de marimbondo $\sim$ idade + tempo de residência + sexo + idade: tempo de residência: sexo; AIC $=181,61)$ foi de $52 \%$, com associação significativa $(p<0,05)$ com idade, tempo de residência e com a interação entre idade, tempo de residência e sexo, mas não com sexo isoladamente. Já GLmabelha (tipos de abelha idade + tempo de residência + idade : tempo de residência; AIC $=157,71$ ) explicou $25 \%$ da variação, com associação significativa apenas com tempo de residência. E a variação explicada por GLmninho (tirou o ninho idade + tempo de residência + sexo; AIC $=61,19$ ) foi de $53 \%$, com associação significativa com tempo de residência. Todos os valores de VIF foram baixos, indicando não haver multicolinearidade entre as preditoras.

Tabela 3. Resumo dos modelos $\mathrm{glm}$ sobre o conhecimento popular sobre marimbondos e abelhas para o número de tipos de marimbondos conhecidos (GLMmarimbondo), o número de tipos de abelhas conhecidas (GLmabelha) e a atitude em relação ao ninho (GLmninho). GLmmarimbondo: tipos de marimbondo idade + tempo de residência + sexo + idade:tempo de residência:sexo (AIC $=181,61)$; GLmabelha: tipos de abelha $\sim$ idade + tempo de residência + idade:tempo de residência (AIC $=157,71)$; e GLmninho: tirou o ninho $\sim$ idade + tempo de residência + sexo $($ AIC $=61,19)$. NA = variável não selecionada para o modelo.Características dos modelos: Distribuição de Poisson para GLmmarimbondo e GLMabelha com função de ligação $\log$, e Distribuição Binomial para e GLmninho com função de ligação logit. * $\mathrm{p}<0,05$; ** $\mathrm{p}<0,01$.

\begin{tabular}{|c|c|c|c|c|c|c|c|c|c|c|}
\hline \multicolumn{2}{|c|}{ Modelos: } & \multicolumn{3}{|c|}{ GLM marimbondo } & \multicolumn{2}{|c|}{ GLMabelha } & \multicolumn{4}{|c|}{ GLM ninho } \\
\hline Identificação da variável & GL & Residuos & $\mathrm{p}$ & $D^{2}$ aj & Residuos & $\mathrm{p} \quad D^{2}$ aj & VIF & Residuos & $\mathrm{p} \quad D^{2}$ aj & VIF \\
\hline Modelo nulo & 49 & 63,92 & & 0,19 & 32,88 & 0,27 & & 62687 & 0,15 & \\
\hline idade & 1 & 2,53 & $* * *$ & & 3,02 & & 1,33 & 0,04 & & 1,38 \\
\hline tempo de residência & 1 & 0,90 & $* * *$ & & 1,71 & * & 1,36 & 6,80 & * & 1,43 \\
\hline sexo & 1 & 0,30 & & & NA & & & 2,70 & & 1,06 \\
\hline idade tempo de residência:sexo & 2 & 8,30 & * & & NA & & & NA & & \\
\hline idade tempo de residência & 2 & NA & & & 3,05 & & & NA & & \\
\hline
\end{tabular}


No GLM marimbondo, a forte influência da idade e do tempo de residência pode ser devido à maior experiência empírica pessoal. Esta pode ser adquirida por meio da combinação destes fatores, ou seja, o aumento da idade coincide com o aumento do tempo vivido na mesma área. Visto que a maioria dos entrevistados sempre morou na região, isso pode influenciar na maior aquisição de conhecimentos sobre hábitos e ambientes habitados por esses himenópteros.

Para GLm abelha e para GLm ninho, a idade não foi determinante, o que pode indicar que o conhecimento sobre abelhas e sobre os riscos da retirada de ninhos pode ter outra fonte além da experiência pessoal, tais como o ensino formal ou os meios de comunicação. Isso porque as abelhas possuem maior visibilidade devido ao valor econômico agregado aos seus produtos, tanto alimentares quanto medicinais. Além disso, esses conhecimentos também podem ser provenientes de moradores mais antigos, repassados oralmente.

\section{CONCLUSÃO}

Os moradores da comunidade de Porto Camargo possuem algum grau de conhecimento sobre vespas sociais e abelhas. Todavia, isso não impede uma postura negativa para com esses insetos, sobretudo para os vespídeos, o que coloca em risco suas populações e inviabiliza o acesso aos serviços ambientais prestados por esses animais. Nessa perspectiva sugere-se a implementação de ações de educação ambiental no sentido de reverter esse quadro e estabelecer condutas conservacionistas para esse patrimônio natural.

\section{AGRADECIMENTOS}

À Prefeitura Municipal de Icaraíma, em especial ao setor de Vigilância Ambiental coordenado pelo servidor Rafael Augusto Martins, pelo apoio durante as atividades de campo. Ao Comitê de Ética em Pesquisa (CEP) do Instituto Federal do Paraná (IFPR), pela aprovação do projeto de pesquisa. Ao graduando em Licenciatura em Ciências Biológicas Giuliano Kaulfuss Bertinoti, pelo auxílio na realização das entrevistas. Aos moradores do distrito de Porto Camargo, por compartilhar as suas percepções e saberes a respeito de vespas sociais e de abelhas. Ao André Bastos da Silva, autor de Silva et al. 2019, que gentilmente compartilhou por e-mail o script que foi modificado para se adequar aos objetivos e aos dados deste trabalho.

\section{REFERÊNCIAS}

Almeida, L.M.; Cavichioli, R.R. Arthropoda. In: Ribeiro-Costa, C.S.; Rocha, R.M. (Org.). Invertebrados: Manual de Aulas Práticas. 2. ed. Ribeirão Preto: Holos, cap. 1, p. 128-130, 2016.

Alves, R.R.N.; Souto, W.M.S. Ethnozoology in Brazil: current status and perspectives. Journal of

Ethnobiology and Ethnomedicine, London. v. 7, n. 22, p. 1-18, 2011.

Alves, R.R.N.; Souto, W.M.S. Ethnozoology: a brief introduction. Ethnobiology and Conservation, v. 4, p. $1-13,2015$.

Alves R.R.N.; Albuquerque, U.P. Ethnozoology: Animals in our lives. 1 st., Academic Press, Elsevier Inc., Amsterdam, 2018.

Athayde, S.; Stepp, J.R.; Ballester, W.C. Engaging indigenous and academic knowledge on bees in the Amazon: implications for environmental management and transdisciplinary research. Journal of Ethnobiology and Ethnomedicine, London, v. 12: 26, 2016.

Barbosa, A.M., Brown, J.A., Jimenez-Valverde, A.; Real, R. modEvA: Model Evaluation and Analysis. R package version 1.3.2., 2016. Disponível em <https://CRAN.R-project.org/package=modEvA > Acesso em: 08 julh. 2020

Bardin, L. Análise de Conteúdo. São paulo: Edições 70, 2011.

Barnosky, A.D.; Matzke, N.; Tomiya, S.; Wogan, G.O.U; Swartz, B.; Quental, T.B.; Marshall, C.; McGuire, J.L.; Lindsey, E.L.; Maguire, K.C.; Mersey, B. \& Ferrer, E.A. 2011. Has the Earth's sixth mass extinction already arrived? Nature, London, v. 471, 51 .

Bertoldi, M.R. Saberes tradicionais como patrimônio cultural imaterial dinamizador do desenvolvimento sustentável. Revista Novos Estudos Jurídicos, Itajaí, v. 19, n. 2, p. 559-584, 2014.

Bomfim, B.L.S.; Filho, I.C. F.; Farias, J.C.; França, S.M.; Barros, R.F.M.; Silva, P.R.R. Etnoentomologia em comunidade rural do cerrado piauiense. Desenvolvimento e Meio Ambiente, Curitiba, v. 39, p. 189-205, 2016.

Bolzani, V., Valli, M., Pivatto, M., \& Viegas, C. Natural products from Brazilian biodiversity as a source of new models for medicinal chemistry. Pure and Applied Chemistry, Berlim, v. 84, n. 9, p. 1837-1846, 2012.

Brasil. Diretoria Técnica de Gestão, Secretaria da Vigilância em Saúde, Ministério da Saúde. Diagnóstico rápido nos municípios para vigilância entomológica de Aedes aegypti no Brasil - LIRAa: metodologia 
para avaliação dos índices de Breteau e Predial. Brasília: Ministério da Saúde; 2005. (Série A - Normas e Manuais Técnicos).

Braghini, F.; Chiapetti, E.; Júnior, J.F.S.; Mileski, J.P.F.; Oliveira, D.F.; Morés, S.; Coelho, A.R.; Tonial, I.B. Qualidade dos méis de abelhas africanizadas (Apis mellifera) e jataí (Tetragonisca angustula) comercializado na microrregião de Francisco Beltrão - PR. Revista de Ciências Agrárias, Lisboa, v. 40, n. 1, p. 279-289, 2017.

Brusca, R.C; Brusca, G.J. Invertebrados. 2. ed. Rio de Janeiro: Guanabara Koogan, 2007. 968 p.

Bueno, E.T.; Souza, M.M.; Clemente, M.A. The Effect of Forest Fragmentation on Polistinae. Sociobiology, Feira de Santana, v. 66, p. 508-514, 2019.

Carvalho, R.M.A.; Martins, C.F.; Mourão, J.S. Meliponiculture in Quilombola communities of Ipiranga and Gurugi, Paraíba state, Brazil: an ethnoecological approach. Journal of Ethnobiology and Ethnomedicine, London, v. 10, n. 3, 2014.

Clark, M.; Tilman, D. Comparative analysis of environmental impacts of agricultural production systems, agricultural input efficiency, and food choice. Environmental Research Letters, v. 12, 064016, 2017.

Clemente M.A; Lange D.; Dátilo W.; Del-Claro K.; Prezoto F. Social wasp-flower visiting guild in less structurally complex habitats are more susceptible to local extinction. Sociobiology, Feira de Santana, v. 60, p. 337-344, 2013.

Costa, E.C.; D’Avila, M.; Cantarelli, E.B.; Murari, A.B. Entomologia Florestal. 2 ed. Santa Maria: Ed. da UFSM, 2011. 244p.

Costa Neto, E.M. Folk taxonomy and cultural significance of "Abeia" (Insecta, Hymenoptera) to the Pankarare, Northeastern Bahia State, Brazil. Journal of Ethnobiology, Boston, v. 18, n. 1, p.1-13, 1998.

Costa Neto, E.M. Conhecimentos e usos tradicionais de animais por uma comunidade afrobrasileira do Parque Nacional Chapada Diamantina, Bahia, Brasil: resultados preliminares. Interciência, Caracas, v. 25, n. 9, p.: 423-431, 2000.

Costa Neto, E.M. Estudos etnoentomológicos no estado da Bahia, Brasil: uma homenagem aos 50 anos do campo de pesquisa. Biotemas, Florianópolis, v. 17, p. 117-149, 2004a.

Costa Neto, E.M. O conhecimento etnoentomológico do cavalo-do-cão (Hymenoptera, Pompilidae) no povoado de Pedra Branca, estado da Bahia, Brasil. Revista Brasileira de Zoociências, Juiz de Fora, v. 6, n. 2, p. 249-260, 2004b.

Costa Neto, E.M. La etnoentomología de las avispas (Hymenoptera, Vespoidea) en el poblado de Pedra Branca, estado de Bahia, Nordeste de Brasil. Boletín de la Sociedad Entomológica Aragonesa, Zaragoza, v. 34, p. $247-262,2004 c$

Costa Neto, E.M.; Santos-Fita, D.; González, R.S. La investigación etnoentomológica y la conservación de la biodiversidad. Boletín de la Sociedad Entomológica Aragonesa, Zaragoza, v. 51, p. 367-369, 2012.

Costa-Neto, E.M. Análise etnossemântica de nomes comuns de abelhas e vespas (Insecta, Hymenoptera) na Terra Indígena Pankararé, Bahia, Brasil. Cadernos de Linguagem e Sociedade, Feira de Santana, v. 14, n. 1, 2013

Delignette-Muller, M.L.; Dutang, C. Fitdistrplus: An R Package for Fitting Distributions. Journal of Statistical Software, Los Angeles, v. 64, n. 4, p. 1-34, 2015. Disponível em <http://www.jstatsoft.org/v64/i04/> Acesso em: 08 julh. 2020

Diegues, A.C.; Arruda, R.S.V.; Silva, V.C.F.; Figols, F.A.B.; Andrade, D. Os Saberes Tradicionais e a Biodiversidade no Brasil (1 Ed.). São Paulo: Ministério do Meio Ambiente, 1999.

Ferreira, M.N.; Ballester, W.C.; Dorval, A.; Costa, R.B. Conhecimento tradicional dos Kaiabi sobre abelhas sem ferrão no Parque Indígena do Xingu, Mato Grosso, Brasil. Tellus, Campo Grande, v. 10, n. 19, p. 129-144, 2010.

Fox, J. e Weisberg, S. An \{R\} Companion to Applied Regression, Third Edition. Thousand Oaks CA: Sage, 2019. Disponível em: <https://socialsciences.mcmaster.ca/jfox/Books/Companion/> Acesso em: 08 julho. 2020

Franco, M.L.P.B. Análise de Conteúdo. 2. ed. Brasília DF: Liber Livro, 2005.

Ghosh, P.; Satpute, N.S. Arthropods: the new alternative medicine for the 21 st century. Innovative Farming, Agartala, v. 1, n. 3, p. 79-84, 2016.

Gullan, P.J.; Cranston, P.S. Insetos: Fundamentos da Entomologia. 5. ed. São Paulo: Editora Roca, 2017, 56-57 pp.

Graça, M.B.; Somavilla, A. Effects of forest fragmentation on community patterns of social wasps (Hymenoptera: Vespidae) in Central Amazon. Austral Entomology, Melbourne, v. 58, p. 657-665, 2018.

Hermes, M.G.; Köhler, A. The flower-visiting social wasps (Hymenoptera, Vespidae, Polistinae) in two areas of Rio Grande do Sul State, Southern Brazil. Revista Brasileira de Entomologia, Curitiba, v. 50, n. 2, p. 268 274, 2006

Instituto Chico Mendes de Conservação da Biodiversidade. Livro Vermelho da Fauna Brasileira Ameaçada de Extinção: Volume VII - Invertebrados. In: Instituto Chico Mendes de Conservação da Biodiversidade. (Org.). Livro Vermelho da Fauna Brasileira Ameaçada de Extinção. Brasília: ICMBio. 727p, 2018.

Lopes, M.; Ferreira, J.B.; Santos, G. Abelhas sem-ferrão: a biodiversidade invisível. Agriculturas, v. 2, n. 4, p. 7-9, 2005. 
Lopes, P.F.M.; Silvano, R.; Begossi, A. Da Biologia a Etnobiologia: Taxonomia e etnotaxiomia, ecologia e etnoecologia. In: R.R.N Alves, W.H.S, Souto, J.S. Moura (Org.), Etnozoologia no Brasil: Importância, status e perspectivas futuras. [s.l: s.n.], Recife, 2010, p. 69-94.

Machado, P.A. O homem e os insetos, passado, presente, futuro. Revista Saúde Pública, São Paulo, v. 21, n. 6, p. 474-479, 1987.

Menezes, C.; Silva, C.I.; Singer, R.B.; Kerr, W.E. Competição entre abelhas durante forrageamento em Schefflera arboricola (Hayata) Merr. Bioscience Journal, Uberlândia, v. 23, Supplement 1, p. 63-69, 2007.

Meyer, D.; Zeileis, A.; Hornik, K. The Strucplot Framework: Visualizing Multi-Way Contingency Tables with ved. Journal of Statistical Software, Los Angeles, v. 17, n. 3, p.1-48, 2006. Disponível em <http://www.jstatsoft.org/v17/i03/> Acesso em: 08 julh. 2020

Modro, A.F.H.; Souza, S.; Aburaya, F.H.; Maia, E. Conhecimento dos moradores do médio Araguaia, Estado do Mato Grosso, sobre a utilidade de produtos de abelhas (Hymenoptera, Apidae). Acta Scientiarum, Maringá, v. 31, p. 421-424, 2009.

Morato, E.F.; Campos, L.A.O. Efeitos da fragmentação florestal sobre vespas e abelhas solitárias em uma área da Amazônia Central. Revista Brasileira de Zoologia, Curitiba, v.17, p. 429-444, 2000.

Nogueira-Neto, P. Vida e criação de abelhas indígenas sem ferrão. São Paulo, Nogueirapis, 445p, 1997.

Oliveira, T.C.T.; Souza, M.M.; Pires, E.P. Nesting habits of social wasps (Hymenoptera: Vespidae) in forest fragments associated with anthropic areas in southeastern Brazil. Sociobiology, Feira de Santana, v. 36, p. 189$196,2017$.

Ollerton, J., Winfree, R.; Tarrant, S. How many flowering plants are pollinated by animals? Oikos, Lund, v. 120, p. 321-326. 2011.

Pádua, D.C.; Souza, M.M.; Brunismann, A.G.; Coelho E.L.; Pires, E. Conhecimento popular sobre vespas sociais (hymenoptera, vespidae) nas comunidades do entorno do refúgio da vida silvestre do rio pandeiros, norte do estado de Minas Gerais. Ethnoscientia, Botucatu, v. 2, p. 1-11, 2017.

Peterson, B.G.; Carl, P. PerformanceAnalytics: Econometric Tools for Performance and Risk Analysis. R package version 2.0.4., 2020. Disponível em: 〈https://CRAN.R-project.org/package=PerformanceAnalytics >. Acesso em: 08 julh. 2020

Petiza, S.; Hamada, N.; Bruno, A.C.; Costa-Neto, E.M. Etnoentomología Baniwa. Boletín de la Sociedad Entomológica Aragonesa, Zaragoza, v. 52, p. 323-343, 2013.

Prezoto, F.; Ribeiro-Júnior, C.; Oliveira-Cortes, S.A.; Elisei, T. Manejo de vespas e marimbondos em ambiente urbano. In: A.S. Pinto; M.M. Rossi; E. Salmeron (orgs.). Manejo de Pragas Urbanas. Piracicaba: Editora CP2, cap. 12, p.123-126, 2007.

Prezoto, F.; Maciel, T.T.; Detoni, M.; Mayorquin, A.Z.; Barbosa, B.C. Pest Control Potential of Social Wasps in Small Farms and Urban Gardens. Insects, v. 10, 192, 2019.

Prodanov, C.C.; de Freitas, E.C. Metodologia do trabalho científico: métodos e técnicas da pesquisa e do trabalho acadêmico. Novo Hamburgo: Editora Feevale, 2ª Ed., p. 274, 2013.

Rêgo, M.M.C.; Albuquerque, P.M.C. Polinização do murici. São Luís: MMA/ EDUFMA, 2006.

Reyes-González, A; Camou-Guerrero, A.; Reyes-Salas, O.; Argueta, A.; Casas, A. Diversity, local knowledge and use of stingless bees (Apidae: Meliponini) in the municipality of Nocupétaro, Michoacan, Mexico. Journal of Ethnobiology Ethnomedicine, London, v. 10, 47, 2014.

Rocha, M.C.L.; Alencar, S. Efeitos dos agrotóxicos sobre as abelhas silvestres no Brasil: proposta metodológica de acompanhamento / Maria Cecília de Lima e Sá de Alencar. - Brasília: Ibama, 88 p., 2012.

Santos, G.M.; Antonini, Y. The traditional knowledge on stingless bees (Apidae: Meliponina) used by the

Enawene-Nawe tribe in western Brazil. Journal of Ethnobiology and Ethnomedicine, London, v. 4:19, 2008.

Santos, A.B. Abelhas nativas: polinizadores em declínio. Natureza on line, Santa Teresa, v. 8, n. 3, p. 103106,2010

Santos, J.R.L.; Thomas, S.E.O.; Dorval, A.; Passa, M.C. A etnoentomologia na comunidade Mata Cavalo de Baixo em Nossa Senhora do Livramento, MT, Brasil. Biodiversidade, Rondonópolis, v. 14, n. 2, p.: 84-92, 2015.

Santos, S.C.; Paz, L.C.; Lima, M.O.A. Prospecção tecnológica sobre métodos de controle do mosquito Aedes aegypti. Cadernos de Prospecção, Salvador, v. 12, p. 105-112, 2019.

Santos, F. M. dos. Análise de conteúdo: a visão de Laurence Bardin. Revista Eletrônica de Educação, São Carlos, v. $6, \quad$ n. $\quad 1, \quad$ p. $383-387, \quad 2012 . \quad$ Disponível em: <http://www.reveduc.ufscar.br/index.php/reveduc/article/view/291>. Acesso em: 05 julh. 2020.

São Paulo (Estado) Secretaria do Meio Ambiente, Coordenadoria de Educação Ambiental. Fauna Urbana. São Paulo: SMA / CEA, Vol. I., 216 p., 2013.

Silva, A.L. Animais medicinais: conhecimento e uso entre as populações ribeirinhas do rio Negro, Amazonas, Brasil. Boletim do Museu Paraense Emílio Goeldi. Ciências Humanas, Belém, v. 3, p. 343-357, 2008.

Silva, W. P.; Paz, J. R. L. Abelhas sem ferrão: muito mais do que uma importância econômica. Natureza On Line, Santa Teresa, v. 10, p. 146-152, 2012. 
Silva, A.B, Barros, R.F.M.; Souto, W.M.S., Soares, R.R., Alencar, N.L., Lopes, C.G.R. "Which fishes do I catch?” Predicting the Artisanal Fishers' Local Knowledge About Target-Species in Brazil. Human Ecology, v. 47, p. 865-876, 2019.

Solís, L.; Casas, A. Cuicatec ethnozoology: traditional knowledge, use, and management of fauna by people of San Lorenzo Pápalo, Oaxaca, Mexico. Journal of Ethnobiology and Ethnomedicine, London, v. 15, n.1, 58, 2019.

Souza, D.L.; Evangelista-Rodrigues, A.; Caldas Pinto, M.S. As abelhas como agentes polinizadores. REDVET. Revista Electrônica de Veterinaria, Málaga, v. 8, n. 3, p. 1-7, 2007.

Souza, M.M.; Silva, M.A.; Silva, M.J.; Assis, N.G.R. Barroso, a capital dos marimbondos: vespas sociais (Hyminoptera: Vespidae) do município de Barroso, Minas Gerais. MG BIOTA, Belo Horizonte, v. 1, n. 3, p.: 24-38, 2008.

Souza, M.M.; Zanuncio, J.C. Marimbondos vespas sociais (Hymenoptera: Vespidae). Universidade Federal de Viçosa, 2012, 79p.

Souza, M.M.; Teofilo-Guedes,G.S.; Milani, L.R.; de Souza, A.S.B.; Gomes, P.P. Social Wasps (Vespidae: Polistinae) from the Brazilian Atlantic Forest. Sociobiology, Feira de Santana, v. 67, n.1, p. 1-12, 2020.

Svolenski, A.C.; Leonhardt, G.; Vivekananda, G.; Oliveira, K.L.; Paes, M.L.N.; Machado, P.R. Plano de Manejo do Parque Nacional de Ilha Grande: Contextualização da Unidade de Conservação. Curitiba: ICMBio, 752 p. 2008. Disponível em: http://www.icmbio.gov.br/portal/images/stories/docs-planos-demanejo/parna_ilha_grande_pm.pdf. Acesso em: 01 ago. 2019.

Symonds, M.R.E.; Moussalli, A. A brief guide to model selection, multimodel inference and model averaging in behavioural ecology using Akaike's information criterion. Behavioral Ecology and Sociobiology, v. 65, n. 1, p. 13-21, 2011.

Schwartz-Filho, D.L.; Laroca, S.; Malkowski, S.R. Abelhas. In: S.B. Mikich; R.S Bérnils. (Org.). Livro Vermelho da Fauna Ameaçada no Estado do Paraná. 1ed. Curitiba: Instituto Ambiental do Paraná, v.1, p. 679-712, 2004

Thompson, H.M. Behavioural effects of pesticides in bees - their potential for use in risk assessment. Ecotoxicology, v. 12, p.: 317-330, 2003.

Ulysséa, M.A.; Hanazaki, N.; Lopes, B.C. Percepção e uso dos insetos pelos moradores da comunidade do Ribeirão da Ilha, Santa Catarina, Brasil. Biotemas, v. 23, n. 3, p. 191-202, 2010.

Zuur, A.F.; Ieno, E.N.; Elphick, C.S. A protocol for data exploration to avoid common statistical problems. Methods in Ecology and Evolution, v. 1, p. 3-14, 2010. 


\section{APÊNDICE I}

(Ficha de questionário pessoal)

\begin{tabular}{|l|}
\hline Nome: \\
\hline Idade: \\
\hline Telefone: \\
\hline Há quantos anos você mora na região? \\
\hline Você sabe a diferença de um marimbondovista: \\
\hline Quais tipos de marimo ( ) Feminino ( ) \\
\hline Quais tipos de Abelhas Qual? \\
\hline Onde os marimbô conhece? \\
\hline Onde as abelhas costumam fazer os ninhos (caixas)? \\
\hline Você já retirou algum tipo de ninho de marimbondo ou abelha? \\
\hline Você já tomou alguma ferroada (picada/mordida) de abelha ou de marimbondo? \\
\hline O que você faz quando toma uma ferroada? Passa alguma coisa? Toma algum \\
\hline Oomédio? \\
\hline Os marimbê usa abelha ou marimbondo para fazer alguma coisa? Remédio? Alimento? \\
\hline
\end{tabular}

